\title{
AROUND MATRIX-TREE THEOREM
}

\author{
YURII BuRMAN AND Boris SHAPIRO
}

\begin{abstract}
Generalizing the classical matrix-tree theorem we provide a formula counting, for a given graph, its subgraphs with a fixed 2-core. We use this generalization to obtain an analog of the matrix-tree theorem for the root system $D_{n}$ (the classical theorem corresponds to the $A_{n}$-case). Several byproducts of the developed technique, such as a new formula for a specialization of the multivariate Tutte polynomial, are of independent interest.
\end{abstract}

\section{Introduction}

Let us first fix some definitions and notation to be used throughout the paper. The main object of our study will be an undirected graph $G$ without multiple edges. It is understood as a subset $G \subset\{\{i, j\} \mid i, j \in\{1,2, \ldots, n\}\}$, where elements of $\{1,2, \ldots, n\}$ are vertices and elements of $G$ itself are edges. Informally speaking, this means that we mark (i.e. distinguish) vertices but not edges of $G$ (except for Section 6 where an edge labeling will be used). Usually we will assume that $G$ contains no loops, i.e. edges $\{i, i\}$. Directed graphs (appearing in Sections 2 and 5 for technical purposes) are subsets of $\{1,2, \ldots, n\}^{2}$. Since a graph is understood as a set of edges, notation $F \subset G$ means that $F$ is a subgraph of $G$.

We will denote by $n=v(G)$ the number of vertices of $G$, by $\# G=e(G)$ the number of its edges, and by $k(G)$ the number of connected components. For every connected component $G_{i} \subset G(i=1, \ldots, k(G))$ it will be useful to consider its Euler characteristics $\chi\left(G_{i}\right)=v\left(G_{i}\right)-e\left(G_{i}\right)$. A connected graph containing no cycles will be called a tree; a disconnected one, a forest. Note that the absence of cycles is equivalent to the equality $\chi\left(G_{i}\right)=1$ for all $i$; if cycles are present then $\chi\left(G_{i}\right) \leq 0$.

We will usually supply edges of the graph $G$ with weights. A weight $w_{i j}=w_{j i}$ of the edge $\{i, j\}$ is an element of any algebra $\mathcal{A}$. For a subgraph $F \subset G$ denote $w(F) \stackrel{\text { def }}{=} \prod_{\{i, j\} \in F} w_{i j}$; call it the weight of $F$. For any set $U$ of subgraphs of $G$ call the expression $Z(U)=\sum_{F \in U} w(F)$ the statistical sum of $U$. (By definition, we assume $w_{i j}=0$ if $G$ contains no edge $\{i, j\}$.)

To a graph $G$ with weighted edges one associates its Laplacian matrix $L_{G}$. It is a symmetric $(n \times n)$-matrix with the elements

$$
\left(L_{G}\right)_{i j}= \begin{cases}-w_{i j}, & i \neq j, \\ \sum_{k \neq i} w_{i k}, & i=j .\end{cases}
$$

Received by the editors November 21, 2005.

2000 Mathematics Subject Classification. Primary 05C50, secondary 05B35.

Key words and phrases. Tutte polynomial, matrix-tree theorem, subgraph count.

Research supported in part by the RFBR grants \# N.Sh.1972.2003.1 and \# 05-01-01012a. 
The Laplacian matrix is degenerate; its kernel always contains the vector $(1,1, \ldots, 1)$. However, its principal $(n-1) \times(n-1)$ minors are generally nonzero and enter into the classical matrix-tree theorem whose first version was proved by G. Kirchhoff in 1847:

Theorem 1 ([8]). Let $T_{G}$ be the set of all (spanning) trees of $G$. Then $Z\left(T_{G}\right)$ is equal to any principal $(n-1) \times(n-1)$ minor of $L_{G}$.

This theorem has numerous generalizations (for a review, see e.g. [1] and the references therein). For our purposes the most important will be the "all-minors" theorem by S. Chaiken [2].

Call a subset $J=\left\{\left(i_{1}, j_{1}\right), \ldots,\left(i_{m}, j_{m}\right)\right\} \subset\{1,2, \ldots, n\}^{2}$ component-disjoint if $i_{p} \neq i_{q}$ and $j_{p} \neq j_{q}$ for every $p \neq q$; denote $\Sigma J \stackrel{\text { def }}{=} \sum_{p=1}^{m}\left(i_{p}+j_{p}\right)$. Fix a numeration of the pairs $\left(i_{p}, j_{p}\right) \in J$ such that $i_{1}<\cdots<i_{m}$, and denote by $\tau_{J}$ the permutation of $\{1,2, \ldots, m\}$ defined by the condition $j_{\tau_{J}(1)}<j_{\tau_{J}(2)}<\cdots<j_{\tau_{J}(m)}$.

A forest $F$ with the vertex set $\{1,2, \ldots, n\}$ is called $J$-admissible if it has $m$ components, and every component contains exactly one vertex from the set $\left\{i_{1}, \ldots, i_{m}\right\}$, and exactly one, from $\left\{j_{1}, \ldots, j_{m}\right\}$ (these two may coincide if the sets intersect). Denote by $\gamma_{F, J}$ a permutation of the set $\{1,2, \ldots, m\}$ such that $i_{p}$ and $j_{\gamma_{F, J}(p)}$ lie in the same component of $F$, for every $p=1,2, \ldots, m$.

For an $n \times n$-matrix $M$ and a component-disjoint set $J$ denote by $M(J)$ the submatrix of $M$ obtained by deletion of the rows $i_{1}, \ldots, i_{m}$ and the columns $j_{1}, \ldots, j_{m}$. For any permutation $\sigma$ denote by $\varepsilon(\sigma)= \pm 1$ its sign (parity).

Theorem 2 ([2]). For any component-disjoint subset $J \subset\{1,2, \ldots, n\}^{2}$ one has

$$
(-1)^{\Sigma J} \operatorname{det}\left(L_{G}\right)(J)=\sum_{F} \varepsilon\left(\tau_{J} \circ \gamma_{F, J}\right) w(F)
$$

where the sum is taken over the set of all $J$-admissible subforests $F$ of $G$.

Theorem 1 is a particular case of Theorem 2 corresponding to the situation when $J$ contains one element only.

Most of this article is devoted to various generalizations of Theorem 1. In Section 2 we consider determinant-like expressions for statistical sums of subgraphs $F \subset G$ with cycles (namely, subgraphs with a given 2-core). In Section 3 we consider the case of subgraphs with vanishing Euler characteristics. Spanning trees of a graph $G$ can be interpreted as irreducible linearly independent subsets of roots in the root system $A_{n}$; in Section 4 we prove an analog of Theorem 1 for the root system $D_{n}$.

Two remaining sections form a sort of appendix to the paper. In Section 5 we give an explicit formula for the number $d(G)$ of orientations of the graph $G$ without sources and sinks (this number enters Theorem 3). In Section 6 we provide a formula for the so called external activity polynomial which is a specialization of the multivariate Tutte polynomial of the graph $G$. The latter is defined as

$$
T_{G}(q, w)=\sum_{m=1}^{n} q^{n} Z\left(U_{m}\right)
$$

(see $[12,16,18]$ for details) where $U_{m}$ is the set of subgraphs of $G$ having $m$ connected components and $w$ is a collection of weights of the edges. The formula we prove 
(Theorem 9) is an alternating sign summation over partitions of the set of vertices of $G$.

In the end of the paper we discuss several open problems related to the main topic.

\section{Graphs with a given 2-core}

Let $G$ be an undirected graph (loops and multiple edges are allowed). The maximal subgraph $G^{\prime} \subset G$ such that every vertex of $G^{\prime}$ is an endpoint of at least two edges or is attached to a loop (that is, there are no "hanging" vertices) is called the 2-core of $G$ and denoted by $\operatorname{core}_{2}(G)$. A graph $G$ is the union of $\operatorname{core}_{2}(G)$ and a forests (possibly none) attached to every vertex of $\operatorname{core}_{2}(G)$.

A graph $G$ is called negative if it contains no loops, no multiple edges, $G=\operatorname{core}_{2}(G)$, and $\chi\left(G_{i}\right)<0$ where $G_{i}, i=1, \ldots, k(G)$ are connected components of $G$. A graph $G$ is called non-positive if all the above is true but $\chi\left(G_{i}\right) \leq 0$. A non-positive graph $G$ is the union of a negative graph $G_{0}$ and several cycles, each cycle forming a separate connected component. We will code this situation as $G=G_{0} \cup 3^{k_{3}} \ldots n^{k_{n}}$ where $k_{s}$ stands for the total number of cycles of length $s$.

For any directed graph $Q$ (with the vertex set $\{1,2, \ldots, n\}$ ) denote by $[Q]$ the corresponding undirected graph. Given a $(n \times n)$-matrix $M$ with entries $a_{i j} \in \mathcal{A}$ define

$$
\langle M, Q\rangle \stackrel{\text { def }}{=} \prod_{(i, j) \in Q} a_{i j}
$$

In particular, if $[Q] \subset G$ where $G$ is a graph without loops or multiple edges, with weights $w_{i j}$ (like in the previous section), then $\left\langle L_{G}, Q\right\rangle=(-1)^{e(Q)} w(Q)$.

A directed graph $Q$ is called regular if the following two conditions are satisfied:

(1) $Q$ contains no sources or sinks, i.e. for every vertex there is at least one incoming and one outgoing edge.

(2) If $Q$ contains a loop (an edge $(i, i))$ or a pair of antiparallel edges (edges $(i, j)$ and $(j, i))$ then they form a separate connected component of $Q$.

If $Q$ is a regular directed graph then $[Q]$ consists of a non-positive graph and several loops and double edges (cycles of length 2), each loop and double edge forming a separate connected component. We will denote this by $[Q]=H \cup 1^{k_{1}} 2^{k_{2}}$ where $H$ is non-positive and $k_{1}, k_{2}$ are the number of loops and double edges, respectively.

In what follows it will be convenient to allow graphs to have multiple (more specifically, double) edges. If $F$ is a graph with multiple edges we will abuse notation writing $F \subset G$ if the graph obtained from $F$ by neglecting the multiplicities is a subgraph of $G$. Computing the weights, we will, however, take multiplicities into account:

$$
w(F) \stackrel{\text { def }}{=} \prod_{\{i, j\} \text { is an edge of } F} w_{i j}^{m_{i j}}
$$

where $m_{i j} \in \mathbb{Z}_{\geq 0}$ is the multiplicity of the edge $\{i, j\}$.

Let $H \subset G$ be a non-positive graph plus several double edges, each double edge forming a separate component. In other words, $H=H_{0} \cup 2^{k_{2}} 3^{k_{3}} \ldots n^{k_{n}}$ where $H_{0}$ is 
negative. Then denote

$$
\varrho_{G}(H)=\sum_{\substack{\Lambda \text { is regular } \\[\Lambda]=H \cup 1^{n-v(H)}}}\left\langle L_{G}, \Lambda\right\rangle
$$

(so that the total number of vertices of $\Lambda$ is $n$ ). By $U(H)$ denote the set of all subgraphs $F \subset G$ such that $\operatorname{core}_{2}(F)=H$.

Theorem 3. Let $H=H_{0} \cup 2^{k_{2}} 3^{k_{3}} \ldots n^{k_{n}}$ be a non-positive graph without loops together with several double edges. Then

$\varrho_{G}(H)=(-1)^{e(H)} d\left(H_{0}\right) \sum_{l_{2}=k_{2}}^{n} \ldots \sum_{l_{n}=k_{n}}^{n}\left(\begin{array}{l}l_{2} \\ k_{2}\end{array}\right) \ldots\left(\begin{array}{c}l_{n} \\ k_{n}\end{array}\right) 2^{l_{3}+\cdots+l_{n}} Z\left(U\left(H_{0} \cup 2^{l_{2}} \ldots n^{l_{n}}\right)\right)$ where $d\left(H_{0}\right)$ is the number of orientations of $H_{0}$ without sources and sinks.

Corollary 1. One has

$$
\begin{aligned}
Z(U(H)) & =(-1)^{e\left(H_{0}\right)} d\left(H_{0}\right) 2^{-\left(k_{3}+\cdots+k_{n}\right)} \times \\
& \times \sum_{l_{2}=k_{2}}^{n} \cdots \sum_{l_{n}=k_{n}}^{n}(-1)^{l_{2}+l_{3}+\cdots+l_{n}}\left(\begin{array}{c}
l_{2} \\
k_{2}
\end{array}\right) \ldots\left(\begin{array}{c}
l_{n} \\
k_{n}
\end{array}\right) \varrho_{G}\left(H_{0} \cup 2^{l_{2}} \ldots n^{l_{n}}\right) .
\end{aligned}
$$

Remark. Corollary 1 is our closest approximation to a "matrix-subgraph" theorem, that is, the best available analog of Theorem 1 for subgraphs of arbitrary structure. Indeed, the left-hand side of (2.2) is the statistical sum over the graphs with a fixed 2 -core (for trees the 2-core is empty), while the right-hand side is a polylinear function of matrix elements of the Laplacian matrix (in the case of trees it was its principal minor). Notice that, unlike Theorem 1, the right-hand side of (2.2) cannot be computed in polynomial time. This is hardly surprising: it is known that the calculation of the Tutte polynomial (and even its value at almost any point of the plane) is a sharp $P$-hard problem (see $[17, \S 9]$ ). Therefore there is no hope to obtain a formula for the statistical sum of connected subgraphs in $G$ with any given number of edges in the form of a determinant or, in general, to get a formula of polynomial complexity in terms of the Laplacian matrix.

Proof of Theorem 3. Let $\Lambda=\Lambda_{0} \cup \Lambda_{1}$ be a regular subgraph of $G$ such that $\left[\Lambda_{0}\right]=H$ and $\left[\Lambda_{1}\right]=1^{n-v(H)}$. Now, $\left\langle L_{G}, \Lambda\right\rangle=\left\langle L_{G}, \Lambda_{0}\right\rangle\left\langle L_{G}, \Lambda_{1}\right\rangle$. Since $\Lambda_{0}$ contains no loops, then $\left\langle L_{G}, \Lambda_{0}\right\rangle=(-1)^{e(H)} w(H)$.

One has $\left(L_{G}\right)_{i i}=\sum_{k \neq i} w_{i k}$, so that the term $\left\langle L_{G}, \Lambda_{1}\right\rangle$ can be represented as the sum of monomials $w_{i_{1} k_{1}} \ldots w_{i_{s} k_{s}}$ where $\left\{i_{1}, \ldots, i_{s}\right\}$ is the vertex set of $\Lambda_{1}$. In other words, $\left\langle L_{G}, \Lambda_{1}\right\rangle=\sum_{\Theta} w(\Theta)$ where $\Theta$ is the directed graph with $[\Theta] \subset G$ satisfying the following property: if $i \in\left\{i_{1}, \ldots, i_{s}\right\}$ then $\Theta$ contains exactly one edge starting from $i$, and if $i \notin\left\{i_{1}, \ldots, i_{s}\right\}$ is a vertex of $\Theta$ then it is a sink (no edge starts from it).

One can easily see that every connected component of $\Theta$ is either a tree such that all its vertices except the root are in $\left\{i_{1}, \ldots, i_{s}\right\}$, or a graph with exactly one cycle with all its vertices in $\left\{i_{1}, \ldots, i_{s}\right\}$. Thus, $\operatorname{core}_{2}\left(\left[\Lambda_{0} \cup \Theta\right]\right)=H_{0} \cup 2^{l_{2}} \ldots n^{l_{n}}$, where $l_{2} \geq k_{2}, \ldots, l_{n} \geq k_{n}$. 
On the other hand, let $F \subset G$ be a subgraph such that $\operatorname{core}_{2}(F)=H_{0} \cup 2^{l_{2}} \ldots n^{l_{n}}$. To identify $F$ with $\left[\Lambda_{0} \cup \Theta\right]$ one has, first, to point out which "1-cycled" connected components of $F$ belong to $\Lambda_{0}$ and which to $\Theta$ - there are $\left(\begin{array}{l}l_{2} \\ k_{2}\end{array}\right) \ldots\left(\begin{array}{l}l_{n} \\ k_{n}\end{array}\right)$ ways to do this. Having this choice made one must orient the 2-core of $F$ without sources and sinks - the number of such orientations being $d\left(H_{0} \cup 2^{l_{2}} \ldots n^{l_{n}}\right)=2^{l_{3}+\cdots+l_{n}} d\left(H_{0}\right)$.

Proof of Corollary 1. One has $\frac{1}{k !} \frac{d^{k}}{d x^{k}}(1-x)^{l}=\sum_{s=0}^{l}\left(\begin{array}{l}s \\ k\end{array}\right)\left(\begin{array}{l}l \\ s\end{array}\right)(-1)^{s} x^{s}=\left(\begin{array}{l}l \\ k\end{array}\right)(1-x)^{l-k}$, and therefore

$$
\sum_{s=0}^{l}(-1)^{s}\left(\begin{array}{l}
s \\
k
\end{array}\right)\left(\begin{array}{l}
l \\
s
\end{array}\right)= \begin{cases}0, & \text { if } k<l \\
1, & \text { if } k=l\end{cases}
$$

The corollary is now straightforward.

\section{Graphs with vanishing Euler characteristics}

Corollary 1 becomes particularly simple if $H$ is a cycle. Namely, if $H=s^{1}$ (a cycle of length $s$ ) then $\lambda_{G}(H)$ is the statistical sum of the set of all connected subgraphs $F \subset G$ having exactly one cycle of length $s$. The "negative part" $H_{0}$ of the graph $H$ is empty which implies $d\left(H_{0}\right)=1$.

Denote by $\Sigma_{n}$ the symmetric group of order $n$ acting on $\{1,2, \ldots, n\}$, and denote by $\mathcal{D}_{n}$ the set of all partitions of $n$. For a permutation $\sigma \in \Sigma_{n}$ having $k_{1}$ cycles of length $1, k_{2}$ cycles of length 2 , etc., denote $D(\sigma) \stackrel{\text { def }}{=} 1^{k_{1}} \ldots n^{k_{n}} \in \mathcal{D}_{n}$. Finally, for any function $f: \mathcal{D} \rightarrow \mathcal{A}$ define the $f$-determinant of an $(n \times n)$-matrix $M$ with entries $a_{i j} \in \mathcal{A}$ by the formula

$$
\operatorname{det}_{f}(L)=\sum_{\sigma \in \Sigma_{n}} f(D(\sigma)) a_{1, \sigma(1)} \ldots a_{n, \sigma(n)} .
$$

Now one has

$$
\begin{aligned}
\varrho_{G}\left(2^{l_{2}} \ldots n^{l_{n}}\right) & =\sum_{D(\sigma)=1^{n-2 l_{2}-\cdots-n l_{n}} 2^{l_{2} \ldots n^{l_{n}}}}(-1)^{n+2 l_{2}+\cdots+n l_{n}}\left(L_{G}\right)_{1, \sigma(1)} \ldots\left(L_{G}\right)_{n, \sigma(n)} \\
& =\operatorname{det}_{\chi_{l_{2}, \ldots, l_{n}}} L_{G},
\end{aligned}
$$

where

$$
\chi_{l_{2}, \ldots, l_{n}}\left(1^{k_{1}} \ldots n^{k_{n}}\right)= \begin{cases}(-1)^{n+2 l_{2}+\cdots+n l_{n}}, & \text { if } k_{2}=l_{2}, k_{3}=l_{3}, \ldots, k_{n}=l_{n} \\ 0, & \text { otherwise }\end{cases}
$$

Thus, Corollary 1 for a cycle takes the following form:

Statement 1. The statistical sum of the set of subgraphs $F \subset G$ having one cycle of length $s \geq 3$ is equal to $\frac{1}{2} \operatorname{det}_{\tau_{s}} L_{G}$, where $\tau_{s}\left(1^{k_{1}} \ldots n^{k_{n}}\right)=(-1)^{n+2 k_{2}+\cdots+n k_{n}} k_{s}$. The statistical sum of the set of subgraphs $F \subset G$ having one cycle of length 2 is $\operatorname{det}_{\tau_{2}} L_{G}$.

This corollary implies the following formula which is the "matrix-tree theorem" for connected subgraphs containing exactly one cycle of any length $s \geq 3$, that is, connected subgraphs $H \subset G$ with $\chi(H)=0$ : 
Corollary 2. Let $U_{G}$ be the set of all connected subgraphs of $H \subset G$ such that $\chi(H)=0$. Then

$$
Z\left(U_{G}\right)=\frac{1}{2} \operatorname{det}_{\mu}\left(L_{G}\right)
$$

where

$$
\mu\left(1^{k_{1}} 2^{k_{2}} \ldots n^{k_{n}}\right)=(-1)^{n+k_{2}+2 k_{3}+\cdots+(n-1) k_{n}}\left(2 k_{2}+k_{3}+\cdots+k_{n}\right)
$$

A finer result concerning graphs $H \subset G$ such that $\chi\left(H_{i}\right)=0$ for any connected component $H_{i}$ of $H(i=1, \ldots, k(H))$ can be obtained using Theorem 2.

For a graph $G$ and a component-disjoint set $J$ denote by $G-J$ the graph obtained from $G$ by deletion of all the edges $\left(i_{p} j_{p}\right)$ where $\left(i_{p}, j_{p}\right) \in J$. Then Theorem 2 implies

Statement 2. Let $G$ be a graph with the vertex set $\{1,2, \ldots, n\}$, without loops and multiple edges, with weights $w_{i j}$ defined for all the edges. Let $J=\left\{\left(i_{1}, j_{1}\right), \ldots,\left(i_{m}, j_{m}\right)\right\}$ be a component-disjoint subset of $\{1,2, \ldots, n\}^{2}$. Then

$$
(-1)^{n} \varepsilon\left(\tau_{J}\right) w_{i_{1} j_{1}} \ldots w_{i_{m} j_{m}} \operatorname{det}\left(L_{G-J}\right)(J)=\sum_{H}(-1)^{k(H)} w(H)
$$

where the sum is taken over the set of all subgraphs $H \subseteq G$ such that every connected component $H_{i}$ of $H$ contains one cycle (that is, $\chi\left(H_{i}\right)=0$ ), the edges $\left\{i_{1}, j_{1}\right\}, \ldots,\left\{i_{m}, j_{m}\right\}$ enter these cycles and vertices $i_{p}$ and $j_{q}$ alternate along the cycle.

Proof. It follows from Theorem 2 that the product $w_{i_{1} j_{1}} \ldots w_{i_{m} j_{m}} \operatorname{det}\left(L_{G-J}\right)(J)$ is equal to the sum of $\pm w_{i_{1} j_{1}} \ldots w_{i_{m} j_{m}} w(F)$ where $F$ runs over the set of subforests of $G-J$ having $m$ components and such that the $p$-th component contains the vertices $i_{p}$ and $j_{\gamma_{F, J}(p)}$; here $\gamma_{F, J}$ is the permutation of $\{1,2, \ldots, m\}$ defined in Section 1 . In other words, $w_{i_{1} j_{1}} \ldots w_{i_{m} j_{m}} \operatorname{det}\left(L_{G-J}\right)(J)$ is equal to the sum of $\pm w(H)$ where $H=F+J$ is the result of addition to $F$ of the edges $\left\{i_{1}, j_{1}\right\}, \ldots,\left\{i_{m}, j_{m}\right\}$. Thus, $H$ is a graph with one cycle in every connected component; all edges $\left\{i_{p}, j_{p}\right\}$ enter the cycles, and vertices $i_{p}$ and $j_{q}$ alternate along the cycle. The connected components of $H$ are in one-to-one correspondence with the cycles of the permutation $\gamma_{F, J}$. The sign of the term $w(F)$ is equal to $(-1)^{n} \varepsilon\left(\tau_{J}\right) \varepsilon\left(\tau_{J} \circ \gamma_{F, J}\right)=(-1)^{n} \varepsilon\left(\gamma_{G, J}\right)$. The permutation $\gamma_{G, J}$ contains $k(H)$ cycles. The sign of any permutation of $\{1,2, \ldots, n\}$ with $k$ cycles equals $(-1)^{n+k}$, and therefore, the total sign is $(-1)^{k(H)}$.

Denote now

$$
Q_{m}=\sum_{\# J=m} w_{i_{1} j_{1}} \ldots w_{i_{m} j_{m}} \operatorname{det}\left(L_{G-J}\right)(J)
$$

where the sum is taken over the set of all component-disjoint subsets $J \subset\{1,2, \ldots, n\}^{2}$ of cardinality $m$. Statement 2 allows to express the generating function for the sequence $Q_{m}$ :

Theorem 4. One has

$$
\sum_{m=1}^{\infty} Q_{m} t^{m}=(-1)^{n} \sum_{H} w(H) \prod_{i=1}^{k(H)}\left((1+t)^{\ell_{i}(H)}-1\right)
$$


where the sum in the right-hand side is taken over the set of all subgraphs $H \subset G$ such that core $_{2}\left(H_{i}\right)$ is a cycle of length $l_{i}(H)$; here $H_{1}, \ldots, H_{k(H)}$ are connected components of $H$.

Proof. By Statement 2 one has that $Q_{m}=\sum_{H} a_{m}(H) w(H)$ where the sum is taken over the set of all subgraphs $H \subset G$ having exactly one cycle in every connected component. The coefficient $a_{m}(H)$ is equal, to $(-1)^{n+k(H)}$ times the number of component-disjoint sets $J=\left\{\left(i_{1}, j_{1}\right), \ldots,\left(i_{m}, j_{m}\right)\right\}$ such that

- $\left\{i_{p}, j_{p}\right\} \in \operatorname{core}_{2}(H)$ for all $p=1, \ldots, m$.

- For every cycle of $H$ there is at least one edge $\left(i_{p} j_{p}\right)$ entering it.

- If a cycle of $H$ has more than one edge $\left(i_{p}, j_{p}\right)$ in it then the vertices $i_{p}$ and $j_{q}$ alternate along the cycle.

This obviously implies that

$$
a_{m}(H)=(-1)^{n+k(H)} \sum_{\substack{m_{1}+\ldots+m_{k(H)}=m \\
m_{1}, \ldots, m_{k(H)} \geq 1}}\left(\begin{array}{c}
\ell_{1}(H) \\
m_{1}
\end{array}\right) \ldots\left(\begin{array}{c}
\ell_{k(H)}(H) \\
m_{k(H)}
\end{array}\right),
$$

and (3.3) follows.

\section{Corollary 3.}

$$
\sum_{m=1}^{\infty}(-1)^{m} Q_{m}=\sum_{H}(-1)^{n+k(H)} w(H)
$$

\section{Linearly independent subsets of the root systems $A_{n}$ and $D_{n}$}

The technique of Section 3 can be used to obtain results on linearly independent subsets of finite root systems, cf. [11].

The set of positive roots $R_{+}\left(A_{n}\right)$ of the reflection group $A_{n}$ consists of vectors $e_{i j}=b_{i}-b_{j}, 1 \leq i<j \leq n$ where $b_{1}, \ldots, b_{n}$ is the standard basis in $\mathbb{C}^{n}$. We will assign to every root $e_{i j} \in R_{+}\left(A_{n}\right)$ its weight $w_{i j} \in \mathcal{A}$ where $\mathcal{A}$ is any algebra. By definition $w_{j i}=w_{i j}$. For any subset $S \subset R_{+}\left(A_{n}\right)$ of positive roots consider a graph $\Gamma(S)$ with the vertices $1, \ldots, n$ such that $\{i, j\}$ is an edge of $\Gamma(S)$ wherever $e_{i j} \in S$. The edge $\{i, j\}$ bears the weight $w_{i j}$. The graph $\Gamma(S)$ is undirected and contains no loops or multiple edges. If $S^{\prime} \subset S$ then $\Gamma\left(S^{\prime}\right)$ is a subgraph of $\Gamma(S)$. We will write $w(S)$ instead of $w(\Gamma(S))$ for short and denote by $L_{S}$ the Laplacian matrix of the graph $\Gamma(S)$.

For a given subset $S \subset R_{+}\left(A_{n}\right)$ one can consider the group $G(S)$ generated by the reflections in the roots $e_{i j} \in S$. The group $G(S)$ is a subgroup of the Weyl group of $A_{n}$, and therefore the space $V=\left\{\sum_{i=1}^{n} x_{i} b_{i} \mid \sum_{i=1}^{n} x_{i}=0\right\} \subset \mathbb{C}^{n}$ is $G(S)$-invariant. $S$ is called irreducible if $V$ is an irreducible representation of $G(S)$.

The following is obvious:

Theorem 5. A set $S \subset R_{+}\left(A_{n}\right)$ is linearly independent if and only if $\Gamma(S)$ contains no cycles. $S$ is irreducible if and only if $\Gamma(S)$ is connected. A linearly independent set $S^{\prime} \subset S$ is maximal (among linearly independent subsets of $S$ ) if and only if $\Gamma\left(S^{\prime}\right)$ is a forest composed of spanning trees of connected components of $\Gamma(S)$. If $S$ is irreducible 
(that is, $\Gamma(S)$ connected) then any maximal linearly independent subset $S^{\prime}$ of $S$ is also irreducible (that is, $\Gamma\left(S^{\prime}\right)$ is a spanning tree of $\Gamma(S)$ ).

Using matroid terminology, one can reformulate Theorem 5 as follows. (See [12, 18] for more detail about matroids.)

Corollary 4. A submatroid of the linear matroid of $\mathbb{C}^{n}$ generated by vectors $e_{i j} \in S$ is isomorphic to the graphical matroid of $\Gamma(S)$.

One can associate a weight $w_{i j}=w_{j i} \in \mathcal{A}$ to every root $e_{i j} \in R_{+}\left(A_{n}\right)$. So, one can consider weights of the root systems and statistical sums of sets of root systems, as was done for graphs in the previous sections. Now the matrix-tree theorem (i.e. Theorem 1) and Theorem 5 imply:

Statement 3. Let $S \subset R_{+}\left(A_{n}\right)$ be irreducible and $T_{S}$ be the collection of all maximal linearly independent subsets of $S$. Then $Z\left(T_{S}\right)$ is equal to (any) principal minor of the Laplacian matrix $L_{S}$.

Consider now a similar question for the reflection group $D_{n}$. Its set $R_{+}\left(D_{n}\right)$ of positive roots consists of the vectors $e_{i j}^{+}=b_{i}-b_{j}$ (the "+"-vectors) and $e_{i j}^{-}=b_{i}+b_{j}$ (the "-"-vectors) for all $1 \leq i<j \leq n$. We associate to every "+"-vector $e_{i j}^{+}$the weight $u_{i j} \in \mathcal{A}$, and to every "-"-vector $e_{i j}^{-}$the weight $v_{i j} \in \mathcal{A}$. Notions of linearly independent, maximal and irreducible subsets $S \subset R_{+}\left(D_{n}\right)$ are defined exactly as in the $A_{n}$-case.

For every set $S \subset R_{+}\left(D_{n}\right)$ consider the graph $\Gamma(S)$ with the vertices $1, \ldots, n$ where the vertices $i$ and $j$ are joined by the edge marked "+" if $e_{i j}^{+} \in S$, and by the edge marked "-_" if $e_{i j}^{-} \in S$. Thus, the graph $\Gamma(S)$ is undirected, contains no loops, and has at most two edges joining every pair of vertices; all its edges are marked by "+" or "_", and if two edges join the same pair of vertices then their marks are different.

A cycle in $\Gamma(S)$ is called odd if it contains an odd number of edges marked "-".

Theorem 6. A set $S \subset R_{+}\left(D_{n}\right)$ is irreducible if and only if $\Gamma(S)$ is connected. $S$ is linearly independent if and only if every connected component of $\Gamma(S)$ is either a tree or a graph with exactly one cycle, and this cycle is odd. If $S$ is irreducible then a linearly independent set $S^{\prime} \subset S$ is maximal if and only if the following holds: if $\Gamma(S)$ contains no odd cycles then $S^{\prime}=S$, otherwise every connected component of $\Gamma\left(S^{\prime}\right)$ is a graph containing exactly one cycle, and this cycle is odd.

This is a $D$-analog of Theorem 5 and it is obvious as well. Our goal in this section is to obtain a $D$-analog of Statement 3 .

Let $J=\left\{\left(i_{1}, j_{1}\right), \ldots,\left(i_{m}, j_{m}\right)\right\} \subset\{1,2, \ldots, n\}^{2}$ be a component-disjoint subset. Denote $S-J^{-} \stackrel{\text { def }}{=} S \backslash\left\{e_{i_{1} j_{1}}^{-}, \ldots, e_{i_{m} j_{m}}^{-}\right\}$.

Theorem 7. One has

$$
\sum_{m=1}^{n} t^{m} \sum_{\# J=m} v_{i_{1} j_{1}} \ldots v_{i_{m} j_{m}} \operatorname{det}\left(L_{S-J^{-}}\right)(J)=(-1)^{n} \sum_{H} w(H) \prod_{i=1}^{k(H)}\left((1+t)^{\ell_{i}^{-}(H)}-1\right) .
$$

Here the internal sum in the left-hand side is taken over the set of all componentdisjoint sets $J \subset\{1,2, \ldots, n\}^{2}$ of cardinality $m$. The sum in the right-hand side is taken over the set of all subsets $H \subset S$ such that every connected component of 
the graph $\Gamma(H)$ contains exactly one cycle. Above we denote by $k(H) \stackrel{\text { def }}{=} k(\Gamma(H))$ the number of these components, and by $\ell_{i}^{-}(H)(i=1, \ldots, k(H))$ the number of "-"-edges entering the cycle in the $i$-th component.

The proof is completely analogous to that of Theorem 4. A required analog of Statement 3 is now:

\section{Corollary 5.}

$$
\sum_{m=1}^{n}(-2)^{m} \sum_{\# J=m} v_{i_{1} j_{1}} \ldots v_{i_{m} j_{m}} \operatorname{det}\left(L_{S-J^{-}}\right)(J)=(-1)^{n} \sum_{F}(-2)^{k(F)} w(F) .
$$

Here the internal sum in the left-hand side is taken over the set of all componentdisjoint sets $J \subset\{1,2, \ldots, n\}^{2}$ of cardinality $m$. The sum in the right-hand side is taken over the set of all maximal linearly independent subsets $F \subset S$. As usual, $k(F)$ is the number of connected components of the graph $\Gamma(F)$.

Proof. This follows directly from Theorems 7 and 6 and the equality

$$
\prod_{i=1}^{k}\left((-1)^{\ell_{i}}-1\right)= \begin{cases}(-2)^{k}, & \text { if all the } \ell_{i} \text { are odd } \\ 0, & \text { if at least one } \ell_{i} \text { is even }\end{cases}
$$

\section{Orientations without sources and sinks}

Let $G$ be an undirected graph with the vertex set $\{1,2, \ldots, n\}$, without loops (multiple edges are allowed). In this section we give a combinatorial description of the number $d(G)$ of directed graphs $Q$ such that $[Q]=G$ and $Q$ has no sources or sinks. (Recall that the number $d(G)$ enters equation (2.1).)

For a set of vertices $P \subset\{1,2, \ldots, n\}$ of $G$ denote by $\langle P\rangle$ the subgraph of $G$ spanned by $P$ (i.e. having $P$ as its vertex set and containing all the edges of $G$ with both endpoints in $P)$. Denote $k(P) \stackrel{\text { def }}{=} k(\langle P\rangle)$ for short and denote by $\mu(P)=$ $e(\langle\{1,2, \ldots, n\} \backslash P\rangle)$, that is, the number of edges in $G$ having both endpoints outside $P$.

Recall that a graph $F$ is called bipartite if one can split its vertices into two groups such that every edge joins two vertices from different groups. Equivalently, this means that every closed path in $F$ contains an even number of edges.

Theorem 8. Assume that $G$ has no isolated vertices. Then the number $d(G)$ of orientations of $G$ without sources and sinks (i.e. such that for every vertex there is at least one incoming and one outgoing edge) is given by the expression

$$
d(G)=\sum_{m=0}^{n}(-1)^{m} \sum_{P: \# P=m,\langle P\rangle \text { is bipartite }} 2^{\mu(P)+k(P)} .
$$

(By assumption, $k(\varnothing)=0$ and $\mu(\varnothing)$ is equal to the total number of edges in $G$.) 
Proof. Fix a set $P$ of vertices, and let $N(P)$ be the number of orientations of $G$ such that every vertex from $P$ is either a source or a sink. Since an edge cannot join two sources or two sinks, one has $N(P)=0$ if $\langle P\rangle$ is not bipartite.

Suppose now that $\langle P\rangle$ is bipartite. Consider the graph $F$ obtained by adding to $\langle P\rangle$ all the edges having one vertex in $P$ and the other outside $P$. Apparently, $k(F)=k(P)$. Since $G$ has no isolated vertices, every connected component of $F$ has 2 orientations such that every its vertex is either a source or a sink. Thus, the total number of ways to orient the edges of $F$ is $2^{k(P)}$. The number of edges of $G$ not belonging to $F$ is $\mu(P)$. These edges can be oriented arbitrarily, and, therefore, $N(P)=2^{\mu(P)+k(P)}$. The statement follows now from the inclusion-exclusion formula.

Corollary 6. The number of orientations of $G$ without sources and sinks is given by

$$
d(G)=\sum_{m=0}^{n}(-1)^{m} \sum_{P: \# P=m} 2^{\mu(P)} \operatorname{chr}_{\langle P\rangle}(2)
$$

where $\operatorname{chr}_{F}(\lambda)$ is the chromatic polynomial of the graph $F$, that is, the number of ways to color its vertices in $\lambda$ colors so that any two adjacent vertices have different colors. (One assumes $\operatorname{chr}_{\langle\varnothing\rangle}=1$.)

Proof. One has $\operatorname{chr}_{F}(2)=2^{k(F)}$ if the graph $F$ is bipartite, and $\operatorname{chr}_{F}(2)=0$ otherwise.

Corollary 7. The number of orientations of $G$ without sources and sinks is given by

$$
d(G)=\sum_{F \subseteq G} 2^{\mu(F)+k(F)}(-1)^{\chi(F)}
$$

where the sum is taken over the set of all subgraphs $F \subseteq G$, and $\mu(F)$ is the total number of edges in $G$ having no common vertices with the edges from $F$.

Proof. A classical result (see e.g. [18] for proof) relates the multivariate Tutte polynomial to the chromatic polynomial:

$$
\operatorname{chr}_{H}(\lambda)=T_{H}(\lambda,-1)=\sum_{\substack{F \subseteq H \\ v(F)=v(H)}} \lambda^{k(F)}(-1)^{e(F)} .
$$

(-1 in the argument of $T_{H}$ means that one takes $w_{i j}=-1$ for every edge $\{i, j\}$ of $H)$. Now by Corollary 6 ,

$$
d(G)=\sum_{m=0}^{n}(-1)^{m} \sum_{\substack{F \subseteq G \\ v(F)=m}} 2^{\mu(F)} 2^{k(F)}(-1)^{e(F)}=\sum_{F \subseteq G} 2^{\mu(F)+k(F)}(-1)^{\chi(F)} .
$$

where $\chi(F)=v(F)-e(F)$ is the Euler characteristics of $F$. 


\section{Multivariable external activity polynomial}

In the previous section we made use of the fact that the chromatic polynomial of a graph is a specialization of its multivariate Tutte polynomial $T(q, w)$. Below we consider another specialization of $T$ which we call the external activity polynomial.

As in Section 2 let $G$ be a graph without loops or multiple edges and with the weights $w_{i j} \in \mathcal{A}$ assigned to its edges. Suppose that $G$ is connected and fix an arbitrary numeration of its edges. Let $T$ be a spanning tree of $G$ and $e$ be an edge not entering $T$. The graph $T \cup e$ has exactly one cycle, and this cycle contains the edge $e$. An edge $e$ is called externally active for $T$ if $e$ is the smallest edge (with respect to the above numeration) in the cycle. The polynomial

$$
C_{G}(w)=\sum_{T \text { is a spanning tree of } G} w(T) \prod_{\{i, j\} \text { is externally active for } T}\left(w_{i j}+1\right) .
$$

will be called the external activity polynomial of $G$. Its specialization (re)appeared recently in the form of the Hilbert polynomial for a certain commutative algebra related to $G$, see [10].

Obviously, the following statement holds:

Statement 4. If $G$ is connected then $C_{G}(w)=Z\left(U_{0}\right)$, where $U_{0}$ is the set of all connected spanning subgraphs of $G$.

Corollary 8 ([12]). If $G$ is connected then $C_{G}(w)=\lim _{q \rightarrow 0} T_{G}(q, w) / q$ where $T_{G}$ is the multivariate Tutte polynomial.

We present another expression for the polynomial $C_{G}$ :

Theorem 9.

$$
C_{G}(w)=\sum_{k=1}^{n}(-1)^{k-1}(k-1) ! \sum_{\{1,2, \ldots, n\}=P_{1} \sqcup \cdots \sqcup P_{k}} \prod_{\{i, j\}: \exists s}\left(w_{i j}+1\right)
$$

where the internal sum taken is over all partitions of the set of vertices into $k \geq 1$ pairwise disjoint subsets, and the product is taken over the set of all edges $\{i, j\}$ of the graph $G$ such that both endpoints ( $i$ and $j$ ) belong to some $P_{s}, s=1, \ldots, k$.

To prove Theorem 9 we need the following technical lemma.

Lemma 1. For any $n \geq 2$ one has

$$
\sum_{k=1}^{n}(-1)^{k-1} \sum_{\{1,2, \ldots, n\}=P_{1} \sqcup \cdots \sqcup P_{k}}\left(p_{1}-1\right) ! \ldots\left(p_{k}-1\right) !=0,
$$

where $p_{i}=\# P_{i}$ is the cardinality of $P_{i}$.

Proof. Denote by $a_{k, n}$ the coefficient at $(-1)^{k-1}(k-1)$ ! in (6.2) and use induction on $n$ to prove the lemma. For $n=2$ one has $a_{1,2}=1$ (the only possible set partition is $\{1,2\}=\{1,2\}$ ) and $a_{2,2}=1$ (the only possible set partition is $\{1,2\}=\{1\} \sqcup\{2\}$ ), so that (6.2) holds. Let now it hold for some $n$. Partitions of the set $\{1,2, \ldots, n+1\}$ fall into two types: either $\{1,2, \ldots, n+1\}=P_{1} \sqcup \cdots \sqcup P_{k} \sqcup\{n+1\}$, or $\{1,2, \ldots, n+1\}=$ 
$P_{1} \sqcup \cdots \sqcup\left(P_{s} \cup\{n+1\}\right) \sqcup \cdots \sqcup P_{k}$, where in both cases $P_{1} \sqcup \cdots \sqcup P_{k}$ is a partition of $\{1,2, \ldots, n\}$. The sum taken over the set partitions of the first type is

$$
\sum_{k=1}^{n}(-1)^{k} \sum_{\{1,2, \ldots, n+1\}=P_{1} \sqcup \ldots \sqcup P_{k} \sqcup\{n+1\}}\left(p_{1}-1\right) ! \ldots\left(p_{k}-1\right) ! 0 !=0
$$

by the induction hypothesis. The sum taken over the set partitions of the second type equals

$$
\begin{aligned}
\sum_{k=1}^{n} & (-1)^{k-1} \sum_{s=1}^{k} \sum_{\{1,2, \ldots, n+1\}=P_{1} \sqcup \cdots \sqcup\left(P_{s} \cup\{n+1\}\right) \sqcup \cdots \sqcup P_{k}}\left(p_{1}-1\right) ! \ldots p_{s} ! \ldots\left(p_{k}-1\right) ! \\
& =\sum_{s=1}^{k}(-1)^{k-1} \sum_{s=1}^{k} p_{s} \sum_{\{1,2, \ldots, n\}=P_{1} \sqcup \cdots \sqcup P_{s} \sqcup \ldots \sqcup P_{k}}\left(p_{1}-1\right) ! \ldots\left(p_{s}-1\right) ! \ldots\left(p_{k}-1\right) ! \\
& =n \sum_{s=1}^{k}(-1)^{k-1} \sum_{\{1,2, \ldots, n\}=P_{1} \sqcup \cdots \sqcup P_{k}}\left(p_{1}-1\right) ! \ldots\left(p_{k}-1\right) ! \\
& =0 .
\end{aligned}
$$

Proof of Theorem 9. Notice first that for any graph $F$ the statistical sum of all subgraphs $H \subset F$ equals

$$
Z(\{H \mid H \subset F\})=\prod_{\{i, j\} \text { is an edge of } F}\left(1+w_{i j}\right) .
$$

Consider the poset $\mathcal{P}_{n}$ of all set partitions of $\{1,2, \ldots, n\}$ ordered by refinement. In particular, $\min =\{1\} \sqcup \cdots \sqcup\{n\}$ is the smallest element of $\mathcal{P}_{n}$, and $\operatorname{Max}=\{1,2, \ldots, n\}$ is its largest element.

Recall (from Section 5) that for a set $P \subset\{1,2, \ldots, n\}$ one denotes by $\langle P\rangle$ the subgraph of $G$ with the vertex set $P$; edges of $\langle P\rangle$ are all the edges of $G$ with both endpoints in $P$. Statement 4 implies now that

$$
Z(\{H \mid H \subset F\})=\sum_{\left(P_{1} \sqcup \ldots \sqcup P_{k}\right) \in \mathcal{P}_{n}} C_{\left\langle P_{1}\right\rangle}(w) \ldots C_{\left\langle P_{k}\right\rangle}(w) .
$$

By Lemma 1 the value $\mu(u, \operatorname{Max})=(-1)^{k-1}(k-1)$ ! where $\mu(u, v)$ is the Möbius function for the poset $\mathcal{P}_{n}$. Therefore theorem follows from the Möbius inversion formula, see e.g. [13].

\section{Questions and final remarks}

Multivariate Tutte polynomial has been studied intensively since 1970s when it was found to be related to partition functions of some important models in mathematical physics (Ising model, Potts model, and more; for more information consult $[5,3]$, references therein and also the review paper [12].) Of particular interest are the complex zeros of these polynomials because they are responsible for the phase transition in ferromagnetic and antiferromagnetic media. It might be very interesting to study the zeros of the external activity polynomial $G_{G}$. Many natural questions 
about them (including the half-plane property, see [12]) are still open. Notice however that by Corollary 8 the polynomial $C_{G}$ is related to the specialization of the Tutte polynomial at $q=0$ while in Potts model $q$ is interpreted as a number of states (of the spin) - we are leaving it to professional physicists to give a sensible interpretation to the polynomial $C_{G}$.

Another possible direction of study is suggested by the nature of formulas (2.1), $(2.2),(3.2),(3.4),(4.1),(5.1),(5.2)$ and $(6.1)$ : they all contain a sign alternating summation. It is highly probable that these formulas present the Euler characteristics of suitable complexes; we pose a problem of finding these complexes. They must be related to the categorification of the Tutte polynomial obtained recently by E.F. JassoHernandez and Y.Rong in [6]; see also [7, 14, 15] where the categorification of the chromatic polynomial was carried out.

Section 3 of this paper contains two different descriptions of the statistical sum of graphs $G$ such that every connected component of $G$ has vanishing Euler characteristics. One is given by the Statement 1 and its corollary (both are special cases of Theorem 3), and the other is contained in Theorem 4 and its corollary, based on the all-minors version of the matrix-tree theorem. The relation between these results resembles the relation between a determinant and its minors decomposition. It seems very interesting to find similar results for graphs with an arbitrary 2-core.

\section{Acknowledgments}

The first named author is sincerely grateful to the Mathematics Department of Stockholm University for the hospitality and financial support of his visit in September 2005 when the essential part of this project was carried out. We are thankful to Professors N. Alon and A. Sokal for their comments on the Tutte polynomial and a number of relevant references. We are grateful to Professor Olivier Bernardi who pointed out an important mistake in an earlier version of this paper.

\section{References}

[1] A. Abdesselam, The Grassmann-Berezin calculus and theorems of the matrix-tree type, Adv. Appl. Math. 33 (2004) 51-70.

[2] S. Chaiken, A combinatorial proof of the all minors matrix tree theorem, SIAM J. Algebraic Discrete Methods 3 (1982), no. 3, 319-329.

[3] S.-Ch. Chang, J.L. Jacobsen, J. Salas and R. Shrock, Exact Potts model partition functions for strips of the triangular lattice J. Statist. Phys. 114 (2004) 763-823.

[4] S. Janson, D.E. Knuth, T. Luczak and B. Pittel, The birth of the giant component, Random Structures Algorithms 4 (1993) 233-358.

[5] C.M.Fortuin, P.W.Kasteleyn, On the random cluster model. I. Introduction and relation to other models, Physica 57 (1972) 536-564.

[6] E.F.Jasso-Hernandez, Y. Rong, A Categorification for the Tutte Polynomial, math.CO/0512613.

[7] L. Helme-Guizon, Y.Rong. A categorification for the chromatic polynomial. Algebr. Geom. Topol. 5 (2005) 1365-1388.

[8] G. Kirchhoff, Über die Auflösung der Gleichungen, auf welche man bei der Untersuchung det linearen Verteilung galvanischer Ströme gefurht wird, Ann. Phys. Chem, 72 (1847) 497-508.

[9] G. Masbaum, A. Vaintrob, A new matrix-tree theorem, Internat. Math. Res. Notices 27 (2002) $1397-1426$.

[10] A. Postnikov and B. Shapiro, Trees, parking functions, syzygies, and deformations of monotone monomial ideals, Trans. Amer. Math. Soc., 356 (2004) 3109-3142. 
[11] A. Postnikov, B. Shapiro, M. Shapiro, Algebras of Curvature Forms on Homogeneous Manifolds Algebras of Curvature Forms on Homogeneous Manifolds, in Infinite-dimensional Lie algebras \& Applic., AMS Trans. Ser. 2, 194 (2000) 227-235.

[12] Alan D. Sokal, The multivariate Tutte polynomial (alias Potts model) for graphs and matroids, math.CO/0503607.

[13] R.P.Stanley, Enumerative Combinatorics, Wadsworth \& Brooks, Monterey, 1986.

[14] M.Stosic, Categorification of the dichromatic polynomial for graphs, math.GT/0504239.

[15] M.Stosic, New categorifications of the chromatic and the dichromatic polynomials for graphs, math.QA/0507290.

[16] W.T. Tutte, A contribution to the theory of chromatic polynomials, Canad J. Math. 6 (1954) $80-91$.

[17] D.J.A. Welsh, The Tutte polynomial, in Statistical physics methods in discrete probability, combinatorics, and theoretical computer science (Princeton, NJ, 1997).

[18] D.J.A. Welsh, C. Merino, The Potts model and the Tutte polynomial, J. Math. Phys. 41 (2000), no. $3,1127-1152$.

Independent University of Moscow, Bolshoi Vlas'Evskit Per. 11, 121002, Moscow, Russia

E-mail address: burman@mccme.ru

Mathematics Department, Stockholm University, S-106 91 Stockholm, Sweden

E-mail address: shapiro@math.su.se 